\title{
Admission for and mortality from primary venous thromboembolism in women of fertile age in Denmark, 1977-95
}

\author{
Lene Mellemkjær, Henrik Toft Sørensen, Lene Dreyer, Jørn Olsen, Jørgen H Olsen
}

Institute of Cancer Epidemiology, Danish Cancer Society, DK-2 100 Copenhagen $\varnothing$, Denmark

Lene Mellemkjær research fellow Lene Dreyer research fellow Jørgen H Olsen head of department

Department of Medicine V, Aarhus University Hospital, DK-8000 Aarhus C, Denmark

Henrik Toft

Sørensen

associate professor

Danish

Epidemiology

Science Centre at

Department of

Epidemiology and

Social Medicine

University of

Aarhus, DK-8000

Aarhus C

Jørn Olsen

professor

Correspondence to:

L Mellemkjær

lene@cancer.dk

BMJ 1999;319:820-1
Epidemiological studies indicate that use of third generation oral contraceptives increases the risk of venous thromboembolism more than does use of second generation oral contraceptives. ${ }^{1-3}$ Critics have suggested that this finding may be confounded by indication, since third generation pills were considered to be safer and were therefore perhaps prescribed more often to women at high risk. If confounding by indication is the only explanation for the observed association, the incidence of venous thromboembolism in the population should not have changed when prescribing patterns changed from second generation to third generation pills with no increase in overall use of oral contraceptives. Vandenbroucke et al and Thomas reported increasing mortality from venous thromboembolism among young women in the Netherlands and in England and Wales from the mid-1980s to the 1990s, when the use of third generation pills was increasing. ${ }^{45}$ Since mortality from venous thromboembolism depends on both aetiological and prognostic factors, however, it may be more appropriate to look at incidence.

In Denmark, roughly a quarter of women aged 15-44 used oral contraceptives during the 1980s and the beginning of the 1990s. The first third generation oral contraceptive, containing desogestrel, was released in 1984, and the third generation preparations containing gestodene and norgestimate were introduced in 1988 and 1990, respectively. Use of third generation pills represented $0.2 \%$ of the total use of oral contraceptives in $1984,17 \%$ in $1988,40 \%$ in 1990 , and $66 \%$ in 1993.

\section{Subjects, methods, and results}

We used admission rates for venous thromboembolism in Denmark as a proxy measure of the incidence of this condition. For people aged 15-49 all admissions during 1977-93 with a registration of pulmonary embolism (ICD-8 (international classification of diseases, 8th revision) code 450.9), deep venous thrombosis (ICD-8 code 451), and other embolic or thrombotic disorders (ICD-8 code 453) were obtained from the Danish National Registry of Patients. Patients were excluded if they had cancer before the venous thromboembolism, surgery within the six months before the venous thromboembolism, or a pregnancy or obstetric diagnosis in the nine months before or three months after the venous thromboembolism or if venous thromboembolism was recorded as a supplemental diagnosis. Multiple admissions for venous embolism for a given person were counted in the numerator of the admission rates provided that the admissions were separated by at least three months. Death rates were calculated from the death certificate file, with no exclusions; during 1994-5 these rates were based on ICD-10 (codes I26, I80, I82). All rates were standardised to the world standard population.

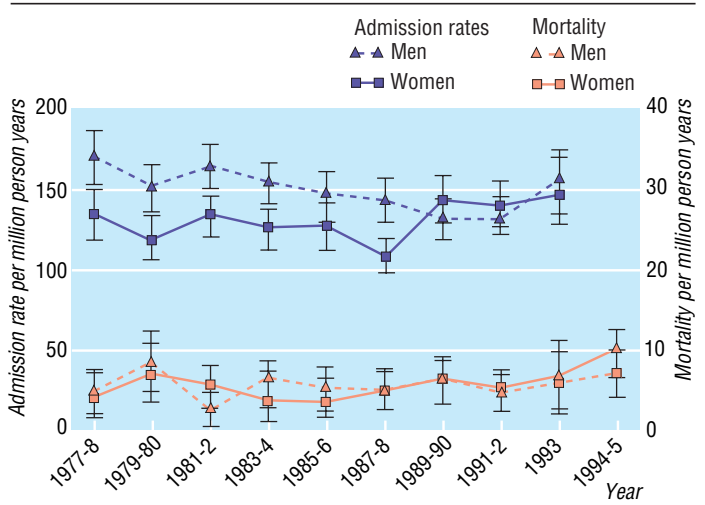

Admission rates and mortality from venous thromboembolism per million person years for men and women aged $15-49$, by calendar year. Rates standardised to world standard population; bars show $95 \%$ confidence intervals

Admission rates for primary venous thromboembolism among women fluctuated around 120 per million person years during $1977-88$ but increased to about 140 per million person years during 1989-93 (figure). For men the admission rates remained stable throughout the period, at a rate similar to that for women in recent years. The mortality curves for men and women followed largely the same course throughout the period. For women the admission rates were based on a total of 2883 discharges with venous thromboembolism during 1977-93, while the death rates were based on only 120 deaths from venous thromboembolism in the same years.

\section{Comment}

The increase in admission rates for venous thromboembolisrn among women seems to correlate with the increase in use of third generation oral contraceptives. The increase is not likely to be explained by changes in diagnostic procedures or in the threshold for admission since no increase was seen for men.

These data support the hypothesis that third generation oral contraceptives increase the risk of venous thromboembolism to a greater extent than other oral contraceptives, and the result cannot be explained by confounding by indication. Our result must, however, be interpreted with caution: the numbers are small, and it is based on data that are subject to misclassification.

We thank Andrea Bautz at the Institute of Cancer Epidemiology, Danish Cancer Society, for programming support.

Contributors: LM initiated the study, discussed core ideas, designed the protocol, and participated in the data analysis and writing of the paper. HTS initiated and coordinated the study, discussed core ideas, and participated in the writing of the paper. LD discussed core ideas, participated in the protocol 
design and data analysis, and edited the paper.JO discussed core ideas and participated in the writing of the paper. JHO discussed core ideas, participated in the protocol design, edited the paper, and is guarantor for the study.

Funding: The study was supported by the Danish Medical Research Council (grant No 9700677). The activities at the Institute of Cancer Epidemiology are financed by the Danish Cancer Society, while the activities at the Danish Epidemiology Science Centre are financed by a grant from the Danish National Research Foundation.

Competing interests: None declared.

1 World Health Organisation Collaborative Study of Cardiovascular Disease and Steroid Hormone Contraception. Venous thromboembolic disease and combined oral contraceptives: results of international multicentre case-control study. Lancet 1995;346:1575-82

2 Jick H, Jick S, Gurewich V, Myers M, Vasilakis C. Risk of idiopathic cardiovascular death and nonfatal venous thromboembolism in women using oral contraceptives with differing progestogen components. Lancet 1995;346:1589-93

3 Spitzer W, Lewis M, Heinemann L, Thorogood M, MacRae K. Third generation oral contraceptives and risk of venous thromboembolic disorders: an international case-control study. BMJ 1996;312:83-8

4 Vandenbroucke J, Bloemenkamp K, Helmerhorst F, Rosendaal F Mortality from venous thromboembolism and myocardial infarction in young women in the Netherlands. Lancet 1996;348:401-2.

5 Thomas S. Mortality from venous thromboembolism and myocardial infarction in young adults in England and Wales. Lancet 1996;348:402.

(Accepted 29 January 1999)

\title{
Association between alcohol consumption and mortality, myocardial infarction, and stroke in 25 year follow up of 49618 young Swedish men
}

\author{
Anders Romelsjö, Anders Leifman
}

Several epidemiological studies have shown that moderate alcohol consumption is associated with reduced mortality from cardiovascular diseases in middle aged and elderly subjects, ${ }^{1}$ but its effect in younger people is unknown. High alcohol consumption is associated with increased total mortality, but the findings for stroke have varied. ${ }^{2}$ We analysed the association between alcohol consumption and the incidence of myocardial infarction, stroke, and mortality in a 25 year follow up of military conscripts.

\section{Subjects, methods, and results}

This study is based on 49618 Swedish men conscripted between 1 July 1969 and 30 June 1970 and born between 1949 and 1952. ${ }^{2}$ At conscription, all men were given two questionnaires with questions covering social background, behaviour, and use of alcohol and tobacco, and all met with a psychologist for assessment. The percentage of non-responders was between $1 \%$ and $2 \%$.

From questions about quantity and frequency of consumption of different alcoholic beverages, we calculated usual consumption in terms of grams of $100 \%$ ethanol a day and categorised subjects into different consumption groups. ${ }^{1}$ Social, psychosocial, and behavioural variables were included as confounders, based on earlier studies and on bivariate analyses of the data in this study (see table). ${ }^{2}$ Using the Swedish personal identification number, we linked the questionnaire data to the Swedish register of causes of deaths and to the new national inpatient care register for 1970-95. We estimated unadjusted and adjusted relative risks in logistic regression analyses and calculated attributable proportions. ${ }^{3}$ Outcomes were total mortality $(\mathrm{n}=1473)$ and incidences of myocardial infarction $(n=279,38$ fatal $)$ and stroke $(n=233,30$ fatal) as underlying causes of death or as main diagnoses at hospitalisation.

Compared with abstainers, alcohol consumers had higher unadjusted relative risks for all three outcomes, and the risks increased with increasing alcohol consumption, being significantly higher for consumers of $\geqslant 15 \mathrm{~g}$ ethanol/day. Adjusted analyses showed an increasing risk of death (significant) and stroke (non-significant) with increasing alcohol consumption but a decreasing risk of myocardial infarction (non-significant) (table). To a considerable extent, the increased mortality with high alcohol consumption was due to the strong association between drinking and smoking and the high risk associated with smoking: compared with not smoking, the relative risk of death was 3.02 for smoking 1-10 cigarettes a day and 5.20 for smoking $\geqslant 11$ cigarettes a day. Consuming $\geqslant 15 \mathrm{~g}$ ethanol/day was associated with a relative risk of death of 1.37 (95\% confidence interval 1.01 to 1.85 ). The attributable proportion in multivariate analysis for alcohol consumption, relative to abstention, was $14 \%$ for mortality (alcohol use caused 205 deaths) and 37\% for stroke (causing 86 cases), while alcohol prevented $44(16 \%)$ myocardial infarctions.

\section{Comment}

The validity of self reported alcohol consumption at conscription and as a measure of consumption during the follow up period can be questioned. ${ }^{3}$ Both non-differential underreporting at conscription and

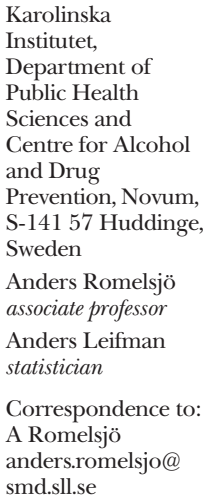

Correspondence to: A Romelsjö anders.romelsjo@ smd.sll.se

BMJ 1999;319:821-2

Relative risks $\left(95 \%\right.$ confidence intervals) ${ }^{*}$ of death, myocardial infarction, and stroke in relation to alcohol consumption in a 25 year follow up of 49618 military conscripts

\begin{tabular}{|c|c|c|c|}
\hline $\begin{array}{l}\text { Alcohol consumption } \\
\text { (g 100\% ethanol/day) }\end{array}$ & $\begin{array}{c}\text { Death } \\
(n=1473)\end{array}$ & $\begin{array}{l}\text { Myocardial infarction } \\
(\mathrm{n}=279)\end{array}$ & $\begin{array}{l}\text { Stroke } \\
(\mathrm{n}=223)\end{array}$ \\
\hline$\overline{0}$ & 1 & 1 & 1 \\
\hline $0.1-14.9$ & 1.13 (0.85 to 1.50$)$ & 0.90 (0.45 to 1.80$)$ & 1.59 (0.64 to 3.92$)$ \\
\hline $15-30$ & 1.32 (0.98 to 1.79$)$ & 0.77 (0.37 to 1.63$)$ & $1.52(0.57$ to 4.00$)$ \\
\hline$\geqslant 30$ & 1.53 (1.08 to 2.16$)$ & 0.61 (0.26 to 1.44$)$ & 2.30 (0.81 to 6.43$)$ \\
\hline$\geqslant 15$ & 1.37 (1.01 to 1.85$)$ & $0.73(0.35$ to 1.52$)$ & 1.70 (0.66 to 4.40$)$ \\
\hline
\end{tabular}

${ }^{*}$ Calculated from logistic regression analysis with adjustments for blood pressure at conscription (continuous), body mass index (weight $(\mathrm{kg}) /(\text { height }(\mathrm{m}))^{2}$ ), father's social class, running away from home, poor school wellbeing, parental divorce, poor emotional control, few (0-1) friends, unemployment for $>3$ months during life, poor health, and smoking as reported at conscription. 